\section{(6) OPEN ACCESS}

\title{
Collagen degradation and neutrophilic infiltration: a vicious circle in inflammatory bowel disease
}

\author{
Pim J Koelink, ${ }^{1}$ Saskia A Overbeek, ${ }^{1}$ Saskia Braber, ${ }^{1}$ Mary E Morgan, ${ }^{1}$ \\ Paul A J Henricks, ${ }^{1}$ Mojtaba Abdul Roda, ${ }^{1}$ Hein W Verspaget, ${ }^{2}$ Simone C Wolfkamp, ${ }^{3}$ \\ Anje A te Velde, ${ }^{3}$ Caleb W Jones, ${ }^{4}$ Patricia L Jackson, ${ }^{5}$ J Edwin Blalock, ${ }^{5}$ \\ Rolf W Sparidans, ${ }^{6}$ John A W Kruijtzer, ${ }^{7}$ Johan Garssen, ${ }^{1}$ Gert Folkerts, ${ }^{1}$ \\ Aletta D Kraneveld ${ }^{1}$
}

- Additional material is published online only. To view please visit the journal online (http://dx.doi.org/10.1136/ gutjnl-2012-303252)

For numbered affiliations see end of article.

\section{Correspondence to} Dr Aletta D Kraneveld, Division of Pharmacology, Faculty of Science, Utrecht Institute for Pharmaceutical Sciences, Utrecht University, PO Box 80082, Utrecht 3508 TB, The Netherlands; A.D.Kraneveld@uu.nl

Received 6 July 2012 Revised 12 February 2013 Accepted 12 February 2013 Published Online First 23 March 2013

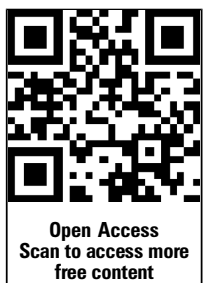

To cite: Koelink PJ, Overbeek SA, Braber S et al. Gut 2014:63. 578-587.

\section{ABSTRACT}

Objective Proline-glycine-proline (PGP) has been shown to have chemotactic effects on neutrophils via CXCR2 in several lung diseases. PGP is derived from collagen by the combined action of matrix metalloproteinase (MMP) 8 and/or MMP9 and prolyl endopeptidase (PE). We investigated the role of PGP in inflammatory bowel disease (IBD).

Design In intestinal tissue from patients with IBD and mice with dextran sodium sulfate (DSS)-induced colitis, MMP8, MMP9 and PE were evaluated by ELISA, immunoblot and immunohistochemistry. Peripheral blood polymorphonuclear cell (PMN) supernatants were also analysed accordingly and incubated with collagen to assess PGP generation ex vivo. PGP levels were measured by mass spectrometry, and PGP neutralisation was achieved with a PGP antagonist and PGP antibodies.

Results In the intestine of patients with IBD, MMP8 and MMP9 levels were elevated, while PE was expressed at similar levels to control tissue. PGP levels were increased in intestinal tissue of patients with IBD. Similar results were obtained in intestine from DSS-treated mice. PMN supernatants from patients with IBD were far more capable of generating PGP from collagen ex vivo than healthy controls. Furthermore, PGP neutralisation during DSS-induced colitis led to a significant reduction in neutrophil infiltration in the intestine.

Conclusions The proteolytic cascade that generates PGP from collagen, as well as the tripeptide itself, is present in the intestine of patients with IBD and mice with DSS-induced colitis. PGP neutralisation in DSS-treated mice showed the importance of PGP-guided neutrophilic infiltration in the intestine and indicates a vicious circle in neutrophilic inflammation in IBD.

\section{INTRODUCTION}

Neutrophils are key inflammatory cells in the innate defence against invading pathogens. The recruitment of neutrophils to the site of inflammation is controlled and directed by the release of endogenous and/or pathogen-derived chemoattractant signals. ${ }^{1}$ Classic endogenous chemoattractants for neutrophils are CXC chemokines such as CXCL8, CXCL1, CXCL2 and CXCL3 in humans and CXCL1 and CXCL2 in mice. ${ }^{2}$ These chemokines induce migration and activation by binding to specific G-protein-coupled receptors on the surface

\section{Significance of this study}

What is already known on this subject?

- Proline-glycine-proline (PGP) is a collagen-degradation product that mediates neutrophil infiltration into the lung via CXCR2 in inflammatory lung diseases such as chronic obstructive pulmonary disease and asthma.

- PGP is formed from collagen by the combined action of matrix metalloproteinase (MMP) 8/9 and prolyl endopeptidase (PE).

- Both collagen proteolysis and neutrophil infiltration are extensively present in inflammatory bowel disease (IBD).

What are the new findings?

- The PGP generation cascade (MMP8/9 and PE) and PGP itself are present in the intestines of patients with IBD and mice with dextran sodium sulfate (DSS)-induced colitis.

- PGP neutralisation reduced neutrophilic infiltration in the intestine of DSS-treated mice.

How might it impact on clinical practice in the foreseeable future?

- Our results show that reducing collagen degradation in the intestine of patients with IBD not only reduces intestinal damage, but also reduces neutrophilic infiltration into the intestine, which opens up new avenues for treating the chronic inflammation found in IBD.

of neutrophils, mainly CXCR1 and CXCR2 in humans and CXCR2 in mice. ${ }^{3}$

Fragments of the extracellular matrix, such as collagen fragments, can also have chemotactic properties. ${ }^{4}$ Recently, a collagen-derived fragment, proline-glycine-proline (PGP), was shown to have chemotactic effects on neutrophils via CXCR2. The tripeptide, PGP, is formed from collagen by the combinational action of matrix metalloproteinases (MMPs) and prolyl endopeptidase (or prolyl oligopeptidase) (PE). ${ }^{6}$ MMP8 and/or MMP9 are responsible for cleavage of collagen into smaller $(<30 \mathrm{kDa})$ fragments, which allow further cleavage by PE. Acetylation of PGP leads to $\mathrm{N}$-acetyl N-Ac-PGP, which is a more potent 
chemoattractant for neutrophils than PGP itself. ${ }^{7}$ The formation of PGP (and N-Ac-PGP) and subsequent neutrophilic infiltration plays an important role in, and is a potential biomarker for, chronic obstructive pulmonary disease and other lung diseases, such as cystic fibrosis. 589

In inflammatory bowel diseases (IBDs), with the clinical manifestations Crohn's disease (CD) and ulcerative colitis (UC), luminal disease activity is accompanied by extensive neutrophilic inflammation. ${ }^{10}$ In addition to the role of chemokines and their receptors in IBD, ${ }^{11} 12$ collagen remodelling and the role of MMPs have also been extensively studied in this disease. ${ }^{13}$ Most of these MMPs show enhanced expression and/or activity in the bowel of patients with IBD. PE is found in all tissues including the intestine. ${ }^{14}$ Similarly, in vivo models of colitis, such as the dextran sodium sulfate (DSS) model, are dependent on proteolytic enzymes. ${ }^{15} 16$ Moreover, blocking CXCR2 with antibodies or CXCR2 antagonists reduces DSS-induced colitis, ${ }^{17}$ and CXCR2 knock-out animals are resistant to DSS-induced colitis. ${ }^{18}$

The aim of the present study was to investigate the components of the collagen-degradation pathway that lead to the production of PGP (MMP8, MMP9 and PE) in IBD. To determine the role of PGP in neutrophilic migration in intestinal inflammation, we investigated these components and the effects of PGP neutralisation in mice with DSS-induced colitis. We hypothesised that, in the bowel of patients with IBD and in mice with DSS-induced colitis, collagen breakdown leads to the production of PGP, enhancing neutrophil chemotaxis to the intestine and exaggerating the disease.

\section{MATERIALS AND METHODS}

\section{Patient samples}

Macroscopically inflamed/affected as well as non-inflamed/ unaffected intestinal mucosa from patients with IBD and colorectal cancer controls was prospectively collected after surgical resection at the Leiden University Medical Centre, The Netherlands. The procedure for homogenising intestinal tissue and methods of assaying myeloperoxidase (MPO) activity and MMP9 protein levels have been reported previously. ${ }^{19}$ The characteristics, MPO activities and MMP9 levels of the subpopulation of patients used here are presented in online supplementary figure S1(A-C). The patients in the polymorphonuclear cell $(\mathrm{PMN})$ study (online supplementary figure S1D) were recruited at the Department of Gastroenterology in the Academic Medical Centre, Amsterdam, The Netherlands. For all studies with human samples, protocols were executed according to the guidelines of the local medical ethics committee and in compliance with the Helsinki Declaration.

\section{Preparation of human samples for PGP measurement}

Intestinal tissue homogenates $(n=60)$ of the same patient resection material were made. Tissue was weighed and homogenised in $1 \mathrm{mM}$ bestatin/phosphate-buffered saline (PBS) (100 mg tissue $/ \mathrm{ml}$ ) using a Precellys 24 tissue homogeniser (Bertin Technologies, France) five times for $10 \mathrm{~s}$ at $6000 \mathrm{rpm}$ with a minimum 5 min cooling period on ice in between. Samples were then centrifuged for $15 \mathrm{~min}$ at $14000 \mathrm{rpm}$, and the supernatant was transferred to a clean tube.

\section{PMN studies and ex vivo PGP generation}

PMNs were isolated from fresh peripheral blood from patients with IBD and healthy controls as previously described, ${ }^{20}$ and incubated $\left(10^{5}\right.$ cells/well) in $100 \mu \mathrm{l}$ RPMI medium 1640 (Lonza Verviers SPRL, Verviers, Belgium) for $6 \mathrm{~h}$. PMNs from healthy controls were also incubated with $10 \mathrm{ng} / \mathrm{ml}$ lipopolysaccharide (LPS) (Sigma-Aldrich Chemie, Zwijndrecht, The Netherlands) for $6 \mathrm{~h}$. A $90 \mu \mathrm{l}$ sample of this PMN-conditioned medium $\left(10^{5}\right.$ cells $/ 100 \mu \mathrm{l} /$ well) was incubated with $10 \mu \mathrm{l}$ of a $1 \mathrm{mg} / \mathrm{ml} \mathrm{solu-}$ tion of type I collagen (Sigma-Aldrich) in PBS for $24 \mathrm{~h}$ at $37^{\circ} \mathrm{C}$ and $5 \% \mathrm{CO}_{2}$ to assess collagen breakdown to (N-Ac-)PGP ex vivo. The collagen was extensively dialysed beforehand to remove PGP.

\section{Measurement of PGP and N-Ac-PGP}

Before peptide measurement, samples were $10 \mathrm{kDa}$-filtered (Millipore). PGP and N-Ac-PGP levels in mouse samples and the ex vivo PGP generation assay were analysed as described previously. ${ }^{5}$ Human intestinal samples were analysed using a Finnigan TSQ Quantum Discovery Max quadrupole mass spectrometer with electrospray ionisation (Thermo Fisher Scientific, San Jose, California, USA) on an Atlantis dC18 column (100 mm $\times 2.1 \mathrm{~mm}$, $\mathrm{d}_{\mathrm{p}}=3 \mu \mathrm{m}$; Waters Chromatography, Milford, Massachusetts, USA) with an Atlantis dC18 pre-column $\left(10 \mathrm{~mm} \times 2.1 \mathrm{~mm}, \mathrm{~d}_{\mathrm{p}}=3 \mu \mathrm{m}\right.$; Waters). Positive electrospray mass transitions were monitored at $270>70,270>116$ and $270>173$ for PGP and $312>140$, $312>112$ and $312>70$ for N-Ac-PGP.

\section{Colitis induction}

Human (hu)CXCR2 C57Bl6 knock-in animals were bred under standard housing conditions with drinking water and food available ad libitum, and genotyped as described previously. ${ }^{21}$ Colitis was induced in male huCXCR2 knock-in mice (8-12 weeks old) by administration of $1.5 \%(\mathrm{w} / \mathrm{v})$ DSS (MP Biomedicals, Aurora, Ohio, USA; molecular mass $=36000-50000 \mathrm{Da}$ ) in tap water to the mice for 5 consecutive days. After a 10-day tap water recovery phase, a second 5-day DSS period was instituted. Mice were killed by $\mathrm{CO}_{2}$ suffocation. All animal studies were approved by the ethics committee for animal studies of Utrecht University.

\section{PGP neutralisation protocols}

The neutralising PGP antagonist, arginine-threonine-arginine (RTR; $300 \mu \mathrm{g} / 100 \mu \mathrm{l} \mathrm{PBS;} \mathrm{Anaspec,} \mathrm{San} \mathrm{Jose,} \mathrm{California,} \mathrm{USA),}$ and polyclonal PGP antibody $(30 \mu \mathrm{g} / 100 \mu \mathrm{l} \mathrm{PBS}$; EZBiolabs, Westfield, Indiana, USA) were administered by intravenous injection into the tail vein, every day from day 0 to day 6 . Control mice were intravenously injected daily with $100 \mu \mathrm{l}$ PBS or $30 \mu \mathrm{g} / 100 \mu \mathrm{l}$ isotype control (rabbit IgG; R\&D Systems, Minneapolis, Minnesota, USA).

\section{Assessment of colitis}

The presence of blood in faeces $(0=$ none, $1=$ positive, $2=$ visible), stool consistency $(0=$ normal, $1=$ loose stools, $2=$ diarrhoea $)$ and general appearance $(0=$ normal, $1=$ hunched back, 2=lethargic) were recorded daily for each animal. Together, these factors constitute the Disease Activity Index (DAI, range 0-6), as described previously. ${ }^{22}$

\section{Histopathology and intestinal tissue sampling}

The large intestines of the killed mice were isolated, and the length was measured in a relaxed position without stretching. The large intestine was opened longitudinally, cleaned in PBS, and longitudinally divided into two pieces. One part was embedded in paraffin (Swiss-role technique), and the other part was stored at $-80^{\circ} \mathrm{C}$ until homogenisation in $1 \mathrm{ml}$ ice-cold PBS as described previously. ${ }^{23}$ Protein concentration was determined using the BCA protein assay kit (Thermo Fisher Scientific). Histology of standard H\&E slides was scored in a blinded fashion by scoring crypt loss and influx of inflammatory cells with a number from 0 (normal) to 4 (severe), and for the 
amount of affected colon $(1,<25 \% ; 2,25-50 \% ; 3,50-75 \%$; $4,75-100 \%)$. The sum of these scores is the histological score (0-16).

\section{Immunohistochemistry}

Immunohistochemistry was performed as described previously. ${ }^{22}$ Antigen retrieval was carried out by boiling the slides in $10 \mathrm{mM}$ citrate buffer ( $\mathrm{pH} 6.0$ ) for $10 \mathrm{~min}$ in a microwave oven. The slides were blocked with 5\% goat serum (Dakocytomation, Glostrup, Denmark) in 1\% BSA in PBS for 20 min at room temperature. The sections were then incubated with primary antibodies (rabbit anti-MMP8 (1:400 dilution; Lifespan Biosciences; LS-C48493), rabbit anti-MMP9 ${ }^{24}$ (1:400 dilution), rabbit anti-PE (1:500 dilution; ProteinTech Group, USA) or rat anti-mouse Ly-6B.2 (1:400 dilution; AbD Serotec, UK; MCA771GA)) in 1\% BSA/PBS overnight at $4^{\circ} \mathrm{C}$. Detection was carried out with biotinylated goat anti-rabbit secondary antibodies (1:200 dilution; Dakocytomation), streptavidin-avidinbiotin complex/horseradish peroxidase (HRP) (Vectastain Elite $\mathrm{ABC}$; Vector Laboratories) and diaminobenzidine tetrahydrochloride (Sigma-Aldrich). Sections were counterstained with Mayer's haematoxylin (Merck), dehydrated and mounted. Slides that had not been incubated with primary antibody were included as negative controls. Photomicrographs were taken with an Olympus BX50 microscope equipped with a Leica DFC 320 digital camera.

\section{Quantification of CXCL1, CXCL2, MMP8, MMP9, leukotriene $\mathrm{B}_{4}\left(\mathrm{LTB}_{4}\right)$ and MPO}

MMP8 (human: R\&D systems; mouse: Cusabio Biotech), $\mathrm{LTB}_{4}$ (R\&D systems) and total and pro-MMP9 (both R\&D Systems) concentrations and MPO activity (Mouse MPO ELISA Kit; Hycult Biotechnology, Uden, The Netherlands) were determined using ELISA kits. The concentration of active MMP9 was calculated by subtracting pro-MMP9 from total MMP9. The levels of CXCL1 and CXCL2 were measured using a mouse multiplex assay according to the manufacturer's instructions (Millipore Corporation, Billericia, Massachusetts, USA). MMP8 and MMP9 levels in PMN-conditioned medium were quantified using a Fluorokine MAP Human Base Kit (R\&D Systems).

\section{Gelatin zymography}

The presence of active and latent forms of MMP9 was analysed by zymography on $11 \%$ polyacrylamide gel containing $1 \%$ porcine skin gelatin (Sigma-Aldrich) as previously described. ${ }^{24}$

\section{Assay of PE activity}

$P E$ activity was measured using the fluorogenic substrate, Z-Gly-Pro-7-amido-4-methylcoumarin (Z-GP-AMC; Bachem, Budendorf, Germany), as described previously. ${ }^{25}$ Tissue homogenate $(20 \mu \mathrm{l} ; 5 \mu \mathrm{g})$ or PMN-conditioned medium $(20 \mu \mathrm{l})$ was incubated with $80 \mu \mathrm{l}$ assay buffer $(100 \mu \mathrm{M}$ Z-GP-AMC in $25 \mathrm{mM}$ Tris/ $\mathrm{HCl}, 0.25 \mathrm{M} \mathrm{NaCl}, \mathrm{pH} 7.5,2 \mathrm{mM} \mathrm{DTT})$ at $37^{\circ} \mathrm{C}$. The fluorescence from released AMC was monitored using a Fluostar reader at an excitation wavelength of $355 \mathrm{~nm}$ and an emission wavelength of $460 \mathrm{~nm}$. Fluorimetric intensities observed were converted into pmol AMC released per minute using appropriate AMC standard curves. PE activity was expressed as $\mathrm{pmol} \mathrm{AMC} / \mathrm{min}$ or $\mathrm{pmol} \mathrm{AMC} / \mathrm{min} / \mathrm{mg}$ protein.

\section{Immunoblotting}

Homogenates were separated on $10 \%(\mathrm{w} / \mathrm{v})$ SDS gels and blotted on to nitrocellulose membranes (Millipore). Membranes were blocked for $1 \mathrm{~h}$ with $5 \%$ milk proteins in PBST (PBS $+0.1 \%$
Tween 20) and subsequently incubated with mouse anti-human MMP8 (1:500 dilution; R\&D systems; MAB9081), rabbit anti-MMP8 (1:500 dilution; Lifespan Biosciences; LS-C48493) or rabbit anti-PE (1:500 dilution; EZBiolabs) in 2\% milk/PBST overnight at $4^{\circ} \mathrm{C}$. Afterwards, membranes were incubated with HRP-labelled secondary antibodies (1:2000 dilution; Dakocytomation) in 2\% milk/PBST, treated with commercial ECL reagents (Amersham Biosciences, Roosendaal, The Netherlands) and finally exposed to photographic film. Blots were then stripped with stripping buffer (Thermo Fisher Scientific) and reprobed with rabbit anti-glyceraldehyde-3-phosphate dehydrogenase (GAPDH; 1:5000 dilution; ITK Diagnostics, Uithoorn, The Netherlands).

\section{Statistical analysis}

Statistical significance of differences between groups was determined by paired $t$ test, unpaired $t$ test, Mann-Whitney $U$ test, Wilcoxon signed rank test, or one-way analysis of variance followed by Dunnett's multiple comparison test using GraphPad Prism 4.0 or SPSS V.16.0. Results were considered significant when $\mathrm{p}<0.05$.

\section{RESULTS}

\section{Protease expression in IBD}

MMP8 levels (figure 1A) were found to be increased in inflamed IBD intestinal tissue homogenates compared with non-inflamed IBD and control tissue, similar to MMP9 levels (see online supplementary figure S1B). ${ }^{19}$ Investigation of MMP8 levels by immunoblotting showed that the majority of the expressed MMP8 is the active form (figure 1C). There were no differences in MMP8 expression in tissue from patients with UC or CD, or in intestinal location (data not shown). There was a strong correlation between MMP8 level (Spearman's $r=0.557, \mathrm{p}<0.001$ ) and MPO activity. Thus, both MMP8 and MMP9, the proteases necessary for the initial collagen-cleavage step, were present in the inflamed bowel specimens obtained from patients with IBD.

\section{PE activity in IBD}

As PE is responsible for cleaving the smaller collagen fragments into PGP, we measured PE activity in the same panel of IBD intestinal tissue homogenates. The activity of PE was similar in control tissue and inflamed and non-inflamed IBD samples (figure 1B). There was also no difference between tissue from patients with UC or CD, or in intestinal location (data not shown). This was confirmed by immunoblotting (figure 1C). In all tissues, a single band was detected at $81 \mathrm{kDa}$, which is the active PE form, as there is no proenzyme form. ${ }^{14}$

\section{Protease expression in the intestine of patients with IBD}

We were interested to determine which cells produce the proteases, MMP8, MMP9 and PE, and if they colocalise within the intestine, enabling generation of PGP from collagen. MMP9 was mainly present in infiltrating immune cells such as neutrophils and macrophages (figure 1D). Weak expression of MMP8 in epithelial cells in addition to expression in inflammatory cells was found in some patient samples (figure 1D), as reported previously. $^{26} \mathrm{PE}$ was expressed in both epithelial cells and inflammatory cells, including neutrophils (figure 1D). To confirm PE production by intestinal epithelial cells, HT29 and Caco 2 cells were cultured in vitro. Both cell lines produced and secreted PE (data not shown).

\section{PGP generation in the intestine of patients with IBD}

Measurement of PGP and N-Ac-PGP in intestinal tissue homogenates showed that N-Ac-PGP levels were significantly higher 
Figure 1 Protease expression in inflammatory bowel disease (IBD). Matrix metalloproteinase 8 (MMP8) protein concentration (A) and prolyl endopeptidase (PE) activity (B) in homogenates of inflamed (infl; $n=50$ ) and non-inflamed (non-infl; $n=42$ ) intestinal tissue from patients with IBD and colorectal cancer controls $(n=20)$. Individual values are shown, and horizontal lines represent mean values. Statistical analysis was by unpaired $t$ test and paired t test. (C) MMP8 and $P E$ immunoblot on control samples (C) and paired non-inflamed (N) and inflamed (I) samples of five patients with IBD, with

glyceraldehyde-3-phosphate dehydrogenase (GAPDH) as a loading control. (D) Immunohistochemistry of MMP8, MMP9 and PE (and negative control) in intestinal tissues of a patient with IBD. The MMP9, PE and negative control shown were on consecutive sections of the same tissue sample. Original magnification $\times 400$.
A

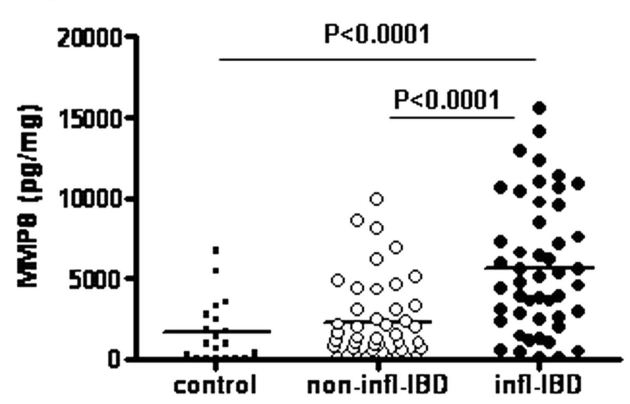

B

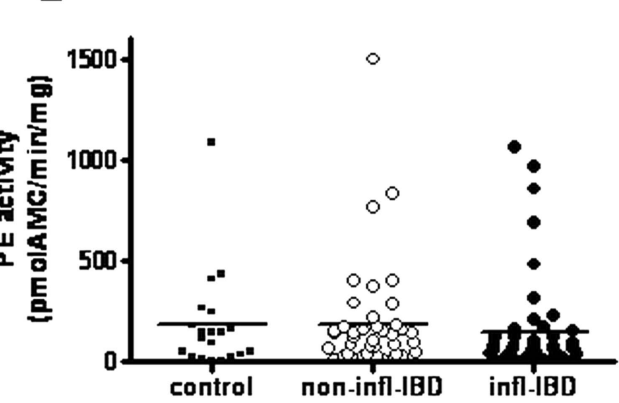

C Marker

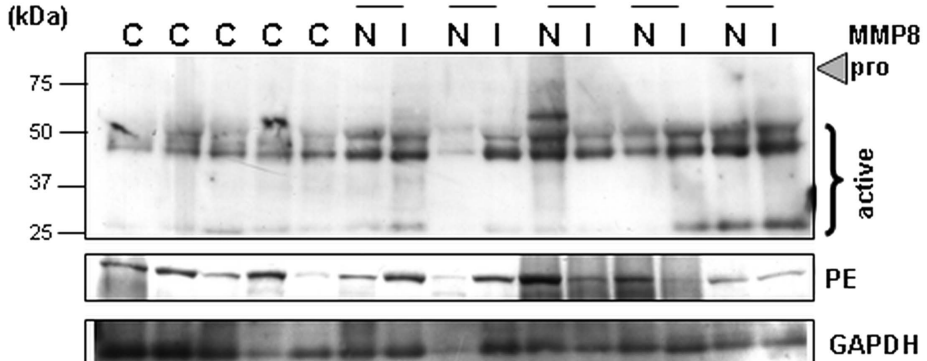

D
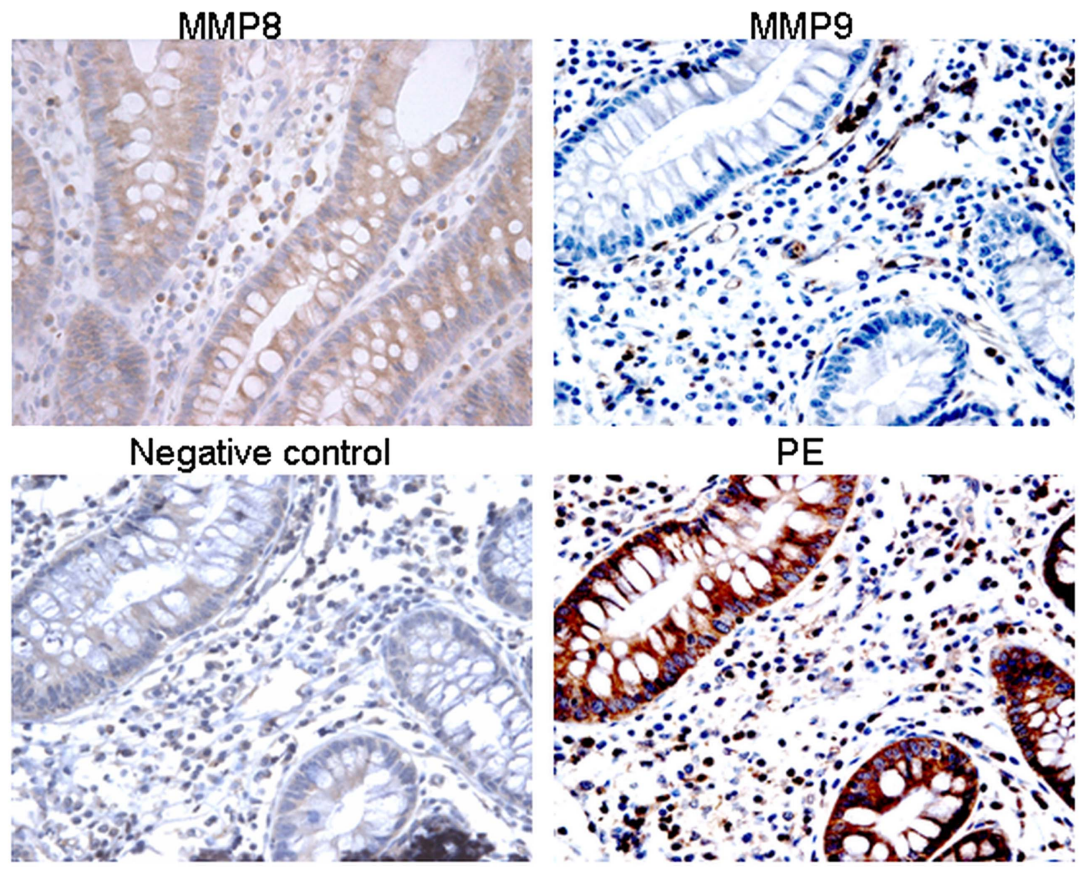

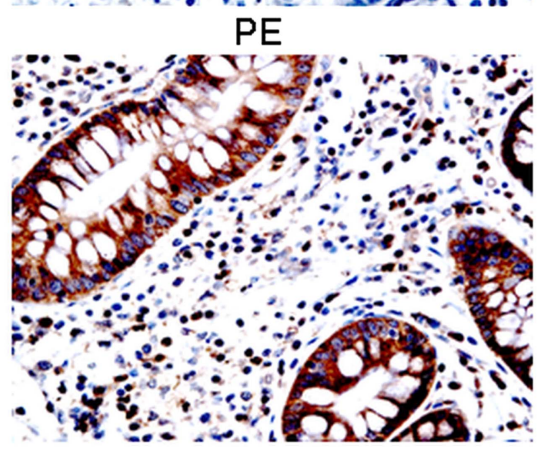

in IBD intestinal tissue samples (figure 2A), while there was a trend towards increased PGP levels (figure 2B). We investigated whether PGP degradation was enhanced in the intestine of patients with IBD. Leukotriene $\mathrm{A}_{4}$ hydrolase $\left(\mathrm{LTA}_{4} \mathrm{H}\right)$ is capable of degrading PGP, ${ }^{27}$ and may also be increased during inflammation. $\mathrm{LTA}_{4} \mathrm{H}$ activity in intestinal tissue homogenates can be measured indirectly by measuring $\mathrm{LTB}_{4}$. There were no differences in $\mathrm{LTB}_{4}$ levels in inflamed versus non-inflamed or control intestinal tissue homogenates (online supplementary figure S2), and therefore no increase in $\mathrm{LTA}_{4} \mathrm{H}$ activity in IBD.

\section{PMNs and PGP generation in IBD}

During inflammation and collagen degradation, MMP8, MMP9 and PE are essential proteases involved in PGP generation, and they can all be produced by neutrophils. ${ }^{28}$ To investigate whether these specific proteases are increased in PMNs from patients with IBD, PMNs isolated from peripheral blood of healthy controls and patients were incubated for $6 \mathrm{~h}$ with medium. Subsequently, MMP8, MMP9 and PE levels were measured in the conditioned medium. Both MMP8 and MMP9 were significantly enhanced in IBD PMNs compared with healthy control PMNs (figure 3A,B), while the released PE activity was similar (figure 3C). This correlates with the data from the tissue homogenates: increased MMP8 and MMP9 at inflamed sites compared with non-inflamed/control samples, and similar PE activity. To investigate if the proteases released by the PMNs were capable of generating (N-Ac-)PGP ex vivo, PMN-conditioned medium was incubated with type I collagen. This showed that PMNs from patients with IBD are much more potent in forming N-Ac-PGP from collagen than healthy control 
Figure 2 Proline-glycine-proline (PGP) and N-Ac-PGP levels in inflammatory bowel disease (IBD). Intestinal N-Ac-PGP (A) and PGP (B) levels in colorectal cancer controls $(\mathrm{n}=5)$ and patients with IBD (inflamed (infl), n=25; non-inflamed (non-infl), $n=18$ ). Non-inflamed and inflamed values were averaged per patient resulting in one intestinal (N-Ac-)PGP value per patient (IBD, $n=27)$. Individual values, expressed as ng (N-Ac-)PGP/g intestinal tissue, are shown, and horizontal lines represent mean values. ${ }^{*} p<0.05$, unpaired $t$ test.
A

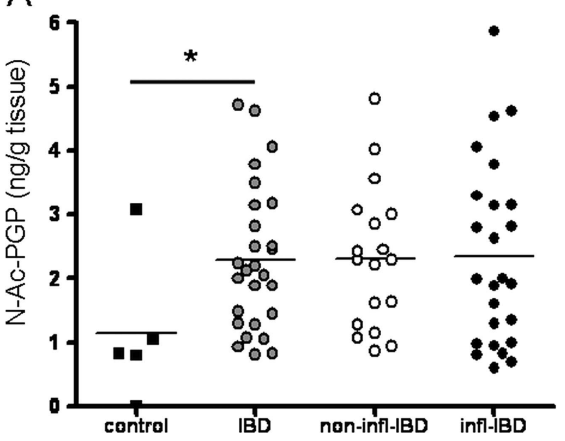

B

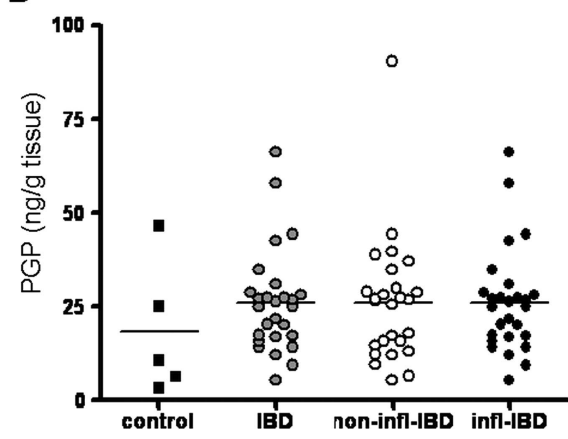

PMNs (figure 3D). PGP levels were also increased, although not significantly (figure 3E). To examine if this difference in neutrophil function was a consequence of the activation state of the neutrophil, PMNs from healthy controls were exposed to the bacterial toxin, LPS. After LPS exposure, PMNs from healthy controls were capable of generating PGP from collagen (online supplementary figure S3). This suggests that, in areas with massive neutrophil infiltration in the intestine of patients with IBD, there is the potential to produce high amounts of (N-Ac-) PGP.

\section{Proteases and PGP generation in the DSS-induced colitis model}

To determine the role of PGP in intestinal neutrophilic inflammation by PGP neutralisation, we investigated the generation of PGP in the DSS-induced colitis model in mice. Because there are species differences in CXCR2-binding characteristics and signalling, we used huCXCR2 knock-in mice ${ }^{21}$ to mimic the human situation more closely.

MMP8, MMP9, PE activity and PGP levels were investigated during two 5-day cycles of $1.5 \%(\mathrm{w} / \mathrm{v})$ DSS with a 10-day normal tap water period in between. The animals had clinicopathological signs of colitis, including moderate body weight loss (online supplementary figure S4A), bloody diarrhoea (online supplementary figure S4B), reduced colon length (online supplementary figure S4C), and increased histopathological scores (online supplementary figure S4D) including increased infiltration of neutrophils, assessed by MPO activity (online supplementary figure S4E), during these cycles. Similar to human IBD, there was no significant increase in intestinal PE activity in the DSS-induced colitis model (figure 4A,B), while MMP9 (both total and active) levels were elevated in the inflamed intestine (figure 4C,D). MMP8 was also slightly elevated (figure 4E) and mainly present in the active form (figure 4F). MMP9 was expressed in infiltrating leucocytes, and MMP8 and PE were found in both infiltrating leucocytes and epithelial cells (figure 5A). As found for human intestinal samples, the generation of both PGP and N-Ac-PGP was evident in intestinal samples (figure 5B,C). There was a strong trend towards increased (N-Ac-)PGP levels in DSS-induced colitis (day 8 vs day $0, \mathrm{p}=0.09$ ).

$\mathrm{LTB}_{4}$ levels also remained unchanged during the first DSS cycle (online supplementary figure S5A). The other neutrophil chemoattractants in mice, CXCL1 and CXCL2, were elevated during the first DSS cycle (online supplementary figure S5B,C), similar to CXCL8 in human IBD. ${ }^{29}$ So the protease expression pattern, (N-Ac)PGP formation and $\mathrm{LTA}_{4} \mathrm{H}$ activity in the DSS-induced colitis model were all similar to that found in IBD patient samples, making this model suitable for studying the effects of (N-Ac-)PGP neutralisation on neutrophil infiltration in the intestine.

\section{Therapeutic intervention by PGP neutralisation}

Because the (N-Ac-)PGP elevation during the first DSS cycle was similar to the elevation found in the intestinal IBD samples, we investigated the effect of PGP neutralisation in this first DSS cycle, by daily treatment with either a PGP-neutralising antibody $^{27}$ or the complementary peptide sequence, arginine-threonine-arginine (RTR). ${ }^{30}$ PGP neutralisation did not have a profound effect on the mild reduction in body weight (data not shown). However, it caused amelioration of wasting of the disease, as judged by a reduction in the other clinicopathological
A

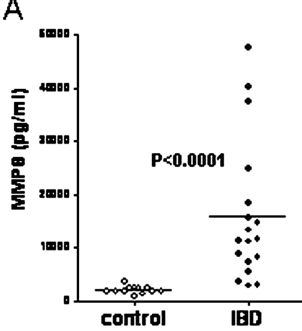

B

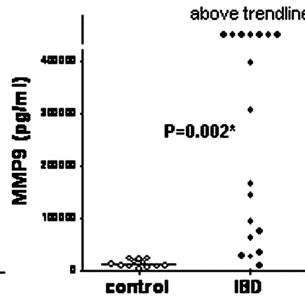

C

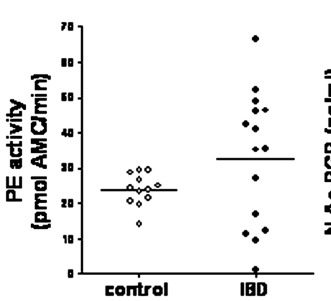

$\mathrm{D}$

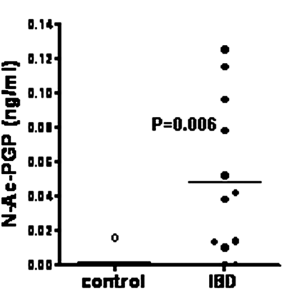

E

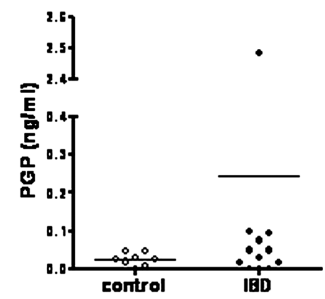

Figure 3 Protease expression and proline-glycine-proline (PGP) generation by polymorphonuclear cells (PMNs) from patients with inflammatory bowel disease (IBD). Matrix metalloproteinase 8 (MMP8) (A) and MMP9 (B) protein levels and prolyl endopeptidase (PE) activity (C) in conditioned medium of PMNs isolated from peripheral blood of patients with IBD $(n=15-20)$ and healthy controls $(n=12)$. N-Ac-PGP (D) and PGP (E) generation from collagen type I by PMN conditioned medium (IBD, $n=12$; control, $n=8$ ). Individual values are shown, and horizontal lines represent mean values. Mann-Whitney tests were used to determine significance. *Values above trendline were not used to calculate significance. AMC, 7-amido-4-methylcoumarin. 
A

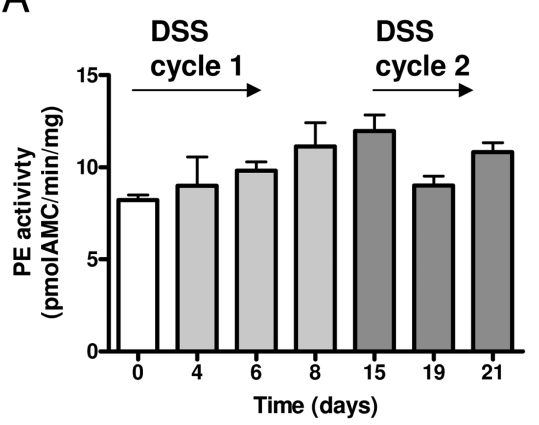

B

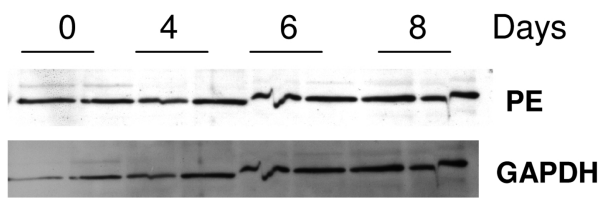

C

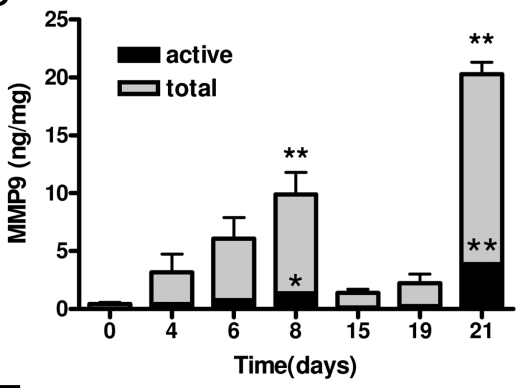

E

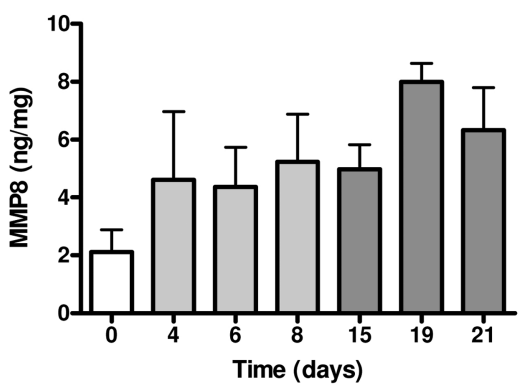

F

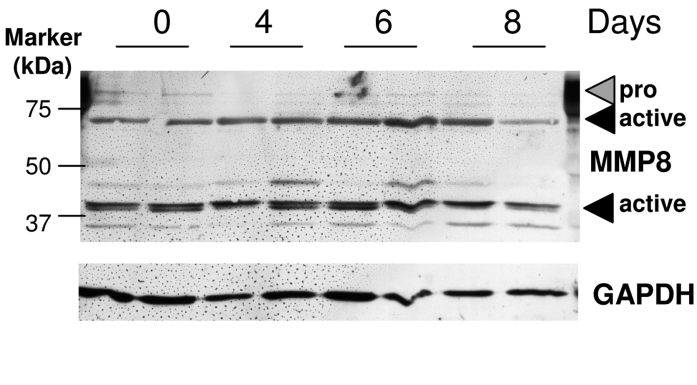

chemoattractants, a collagen-derived tripeptide, PGP, also plays an important role in neutrophilic inflammation in IBD. PGP is generated from collagen by the combined action of MMP8, MMP9 and PE. Our studies identified a previously unrecognised role of these proteases in IBD.

We found that MMP8 and MMP9 are mainly expressed by inflammatory cells in the intestine, and levels of both are increased in IBD, similar to results of others. The other protease involved in the PGP generation cascade, ${ }^{6} \mathrm{PE}$, may be involved in peptide degradation during nutrient absorption in the gut. ${ }^{14}$ To our knowledge, this is the first report of an investigation of $\mathrm{PE}$ activity and protein levels in the intestine of patients with IBD. PE expression and activity were found in the intestine of patients with IBD as well as in control tissue, but there was no difference in inflamed versus non-inflamed or control. In contrast with MMP8 and MMP9, PE was mainly expressed by epithelial cells.

If MMP8 or MMP9 activity is the limiting step in the generation of PGP, one would expect more PGP generation during intestinal inflammation, as PE is already present in healthy intestinal tissue and MMP8 and MMP9 are greatly increased during intestinal inflammation. We indeed found a significant increase

Figure 4 Proteases in dextran sodium sulfate (DSS)-induced colitis. Intestinal prolyl endopeptidase (PE) activity (A) of huCXCR2 knock-in mice treated with 2-day cycles of DSS over time confirmed by immunoblot with glyceraldehyde-3-phosphate dehydrogenase (GAPDH) as a loading control (B). Active and total matrix metalloproteinase 9 (MMP9) levels (C) were confirmed by zymography (D), and MMP8 levels (E) were confirmed by immunoblot with GAPDH as a loading control (F). All values are mean+SEM $(n=3-8)$. ${ }^{*} p<0.05$ versus day 0 (control); ${ }^{* *} p<0.01$ versus day 0 . Statistical analysis was by one-way analysis of variance followed by Dunnett's multiple comparison test. AMC, 7-amido-4-methylcoumarin.

characteristics of DSS-induced colitis, compared with their respective controls (PBS or isotype antibody treatment) (figure 6A). There were no effects of PGP neutralisation on control (water-treated) animals (data not shown). Both the DAI and the shortening of the colon (figure $6 \mathrm{~B}$ ) were significantly reduced at day 7 . Histopathological analysis revealed a significant decrease in the histopathological scores due to PGP neutralisation (figure 6C,D). Moreover, investigation of neutrophil infiltration by immunohistochemistry (figure 6E) and MPO levels (figure $6 \mathrm{~F}$ ) showed that PGP neutralisation significantly reduced neutrophil infiltration in the colon of DSS-treated mice.

The levels of CXCL1 and CXCL2 (online supplementary figure S6A,B) and total and active MMP9 levels (online supplementary figure $\mathrm{S} 7 \mathrm{~A}, \mathrm{~B}$ ) in the intestine were decreased by PGP neutralisation in DSS-induced colitis.

\section{DISCUSSION}

Neutrophils are rapidly recruited to sites of infection in response to $\mathrm{ELR}^{+}$CXC chemokines, such as CXCL8 in humans and CXCL1 and CXCL2 in mice. ${ }^{2}$ In IBD and DSS-induced colitis, these chemokines are elevated in the intestine. ${ }^{11} 12 \mathrm{We}$ now show that, besides these classic neutrophil 
Figure 5 Protease expression and proline-glycine-proline (PGP) levels in dextran sodium sulfate (DSS)-induced colitis. (A) Immunohistochemistry of intestinal matrix metalloproteinase 8 (MMP8), MMP9 and prolyl endopeptidase (PE) (and negative control) on consecutive intestinal sections of a DSS-treated huCXCR2 knock-in mouse. Original magnification $\times 400$. Intestinal N-AC-PGP (B) and PGP (C) levels in DSS-induced colitis, expressed as ng (N-Ac-)PGP/g intestinal tissue. All values are mean + SEM $(n=3-8)$. No significant differences were detected, although there was a strong trend towards increased N-Ac-PGP levels at day 8 (versus day $0, p=0.09$, unpaired t-test).
A

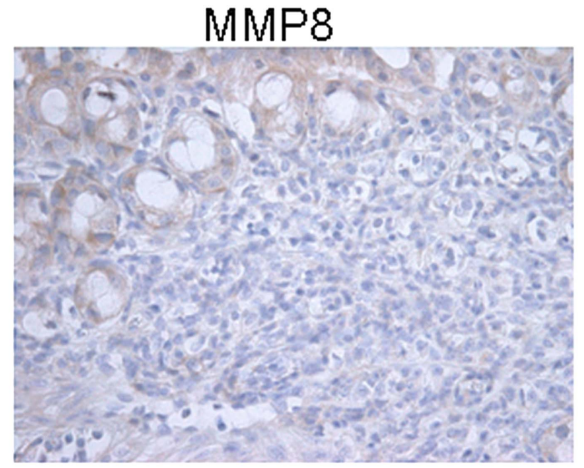

Negative control

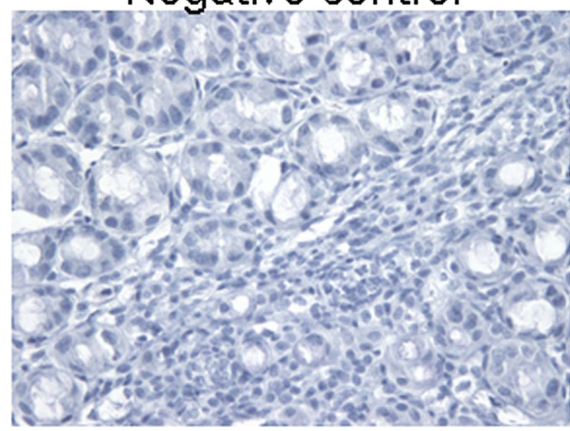

B

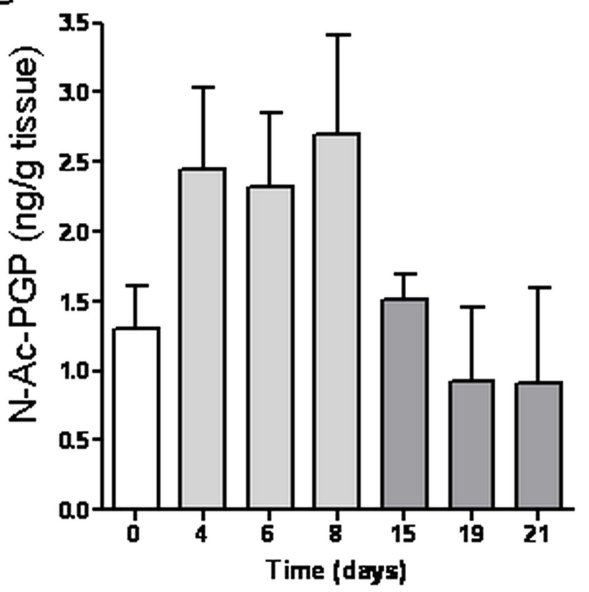

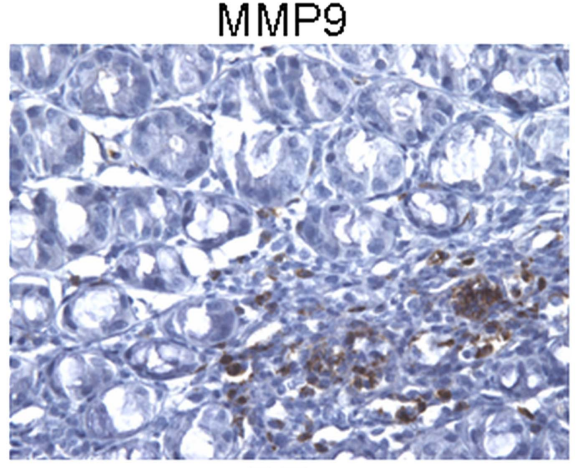

PE

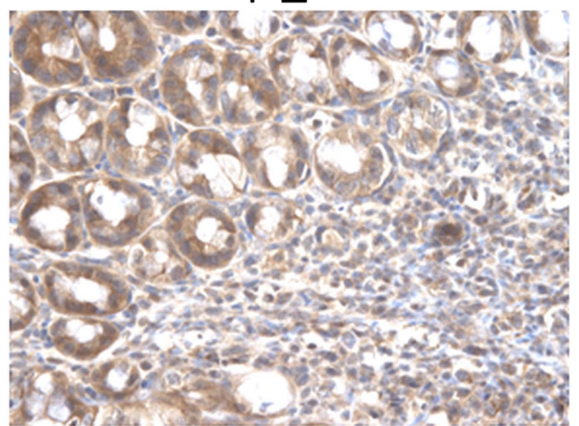

C

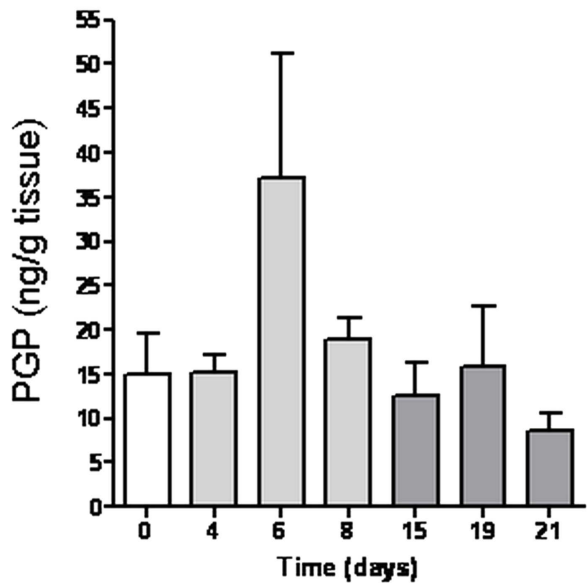

in N-Ac-PGP in intestinal tissue from patients with IBD, and there was also a trend towards increased PGP levels. Collagen type $I$ is the type of collagen that is most abundantly found in the intestine. ${ }^{31}$ Human neutrophils can contain MMP8, MMP9 and PE and are capable of generating PGP from collagen type I on activation. ${ }^{28}$ Because circulating neutrophils from patients with IBD are more activated, ${ }^{32}$ we hypothesised that PMNs isolated from peripheral blood of such patients release more proteases and are more capable of generating PGP from collagen. Indeed we found that PMNs from patients with IBD release more MMP8 and MMP9 and have similar PE levels under unstimulated conditions than PMNs from healthy controls. Moreover, conditioned medium from PMNs obtained from patients with IBD are capable of producing PGP from collagen under unstimulated conditions, in contrast with conditioned medium from PMNs obtained from healthy controls. Activating healthy control PMNs with LPS led to increased MMP8, MMP9 and PE release from healthy control PMNs and PGP generation from collagen, characteristics similar to those of
IBD patient PMNs. As PE is mainly expressed by epithelial cells, high concentrations of PGP could be produced in the epithelium, which in turn results in a chemotactic gradient. As a result, the neutrophils are directed towards the damaged epithelium, where commensal bacteria are triggering the intestinal wall.

$\mathrm{LTB}_{4}$, produced by $\mathrm{LTA}_{4} \mathrm{H}$, is a powerful chemoattractant for neutrophils and is implicated in several chronic inflammatory disorders. ${ }^{33}$ LTA $_{4} \mathrm{H}$ has recently been shown to be capable of degrading PGP, thereby acting as a both a proinflammatory and anti-inflammatory enzyme. ${ }^{27}$ Enhanced expression of $\mathrm{LTB}_{4}$ in intestinal mucosa from patients with IBD has been reported. ${ }^{34}$ LTA $_{4} \mathrm{H}$ activity in inflamed intestinal tissue may therefore be involved in limiting PGP levels. The intestinal levels of $\mathrm{LTB}_{4}$ in the patients with IBD in this study were similar to those in colorectal cancer controls, which indicates that there was no increase in $\mathrm{LTA}_{4} \mathrm{H}$ activity in IBD.

N-Ac-PGP is a far more potent chemoattractant for neutrophils than PGP. ${ }^{7}$ Incubation of IBD PMN-conditioned medium 
A

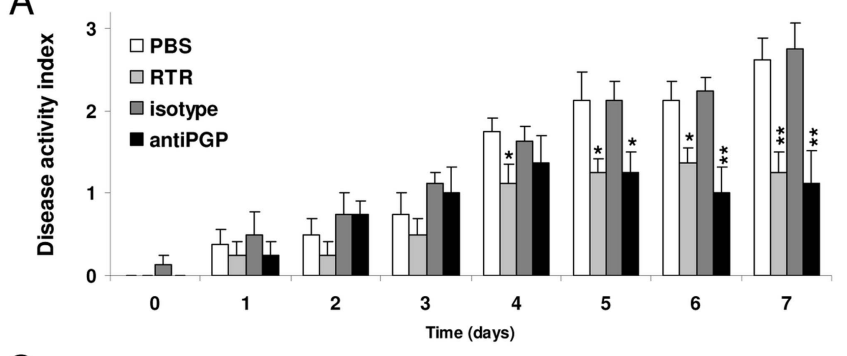

B

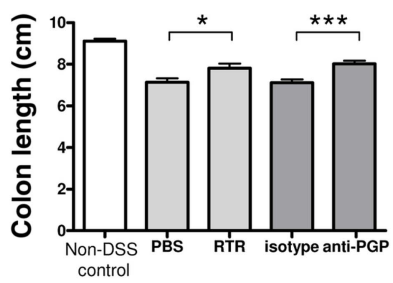

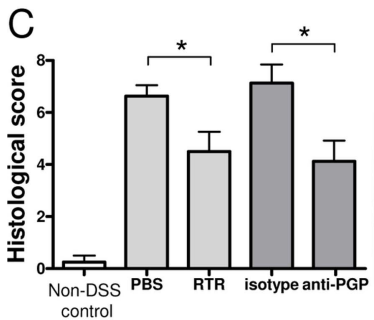

E

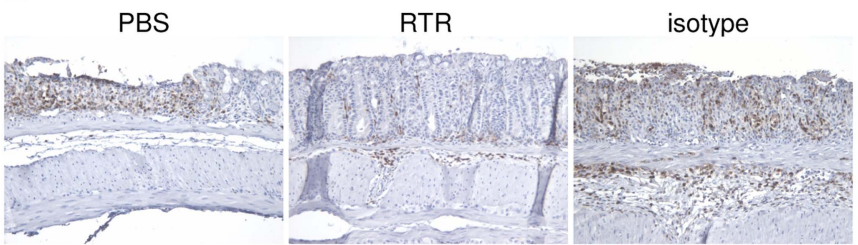

Non-DSS control

PBS

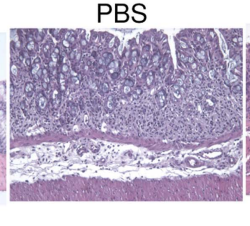

RTR
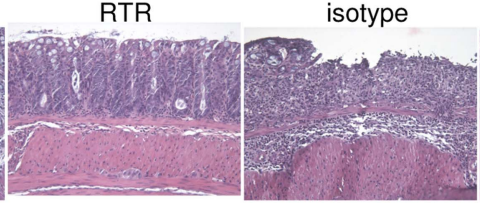

anti-PGP

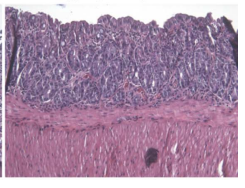

$\mathrm{F}$
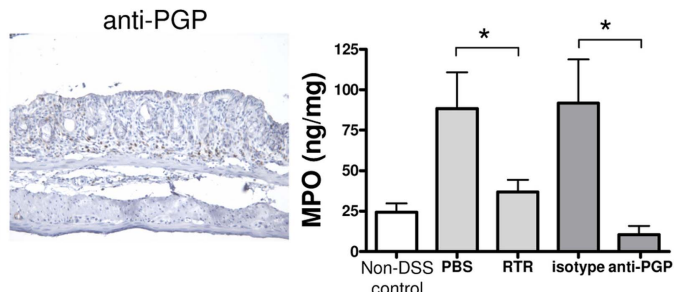

Figure 6 Proline-glycine-proline (PGP) neutralisation in dextran sodium sulfate (DSS)-induced colitis. The Disease Activity Index (A) and colonic length (B) of huCXCR2 knock-in mice treated with 1.5\% (w/v) DSS for 5 days, and daily intravenous injections of arginine-threonine-arginine (RTR) with phosphate-buffered saline (PBS) as control, or PGP antibody with isotype antibody as control, and non-DSS controls. (C) Histopathological scoring and (D) representative images of H\&E-stained intestinal sections of all groups. (E) Representative images of neutrophil (LY-6B.2) immunohistochemistry on intestinal sections of DSS-treated animals treated with RTR, PBS, PGP antibody and isotype antibody. (F) Myeloperoxidase (MPO) levels in intestinal tissue homogenates of all groups as a measurement of neutrophil infiltration. Original magnifications $\times 200$. All values are mean+SEM $(n=8)$. Significance of differences determined by two-tailed Student $t$ test: ${ }^{*} p<0.05 ;{ }^{* *} p<0.01,{ }^{* * *} p<0.001$.

resulted mainly in N-Ac-PGP formation from collagen type I, indicating that IBD neutrophils also release the enzyme necessary for acetylation of PGP. Recent work by our group shows that the PMNs isolated from peripheral blood from patients with IBD (also used in this study) have increased migratory capacities towards CXCL8 compared with healthy control PMNs, probably due to the increased expression of CXCR1 and CXCR2 (Overbeek et al, unpublished work). Therefore PGP levels, and especially N-Ac-PGP levels, may play an important role in neutrophilic infiltration in IBD, as neutrophils from patients with IBD may be more strongly attracted to (N-Ac-) PGP.

To study the pathophysiological role of PGP in neutrophilic inflammation in the colon, we used DSS-induced colitis in mice. This widely used IBD model depends largely on MMP9 protease activity, as MMP9-deficient mice are resistant to DSS-induced colonic inflammation, ${ }^{15}$ and MMP inhibition reduced clinical features. ${ }^{16}$ With regard to the other components involved in the PGP generation cascade, there were striking similarities to human IBD. There was an increase in active MMP8 in colonic tissue from DSS-treated mice, and PE was present. There was a strong trend towards increased (N-Ac-) PGP levels. DSS-induced colitis is characterised by a large CXCR2-dependent influx of neutrophils ${ }^{17}{ }^{18}$ and is therefore highly suitable for studying the role of PGP in neutrophilic infiltration in the intestine. Neutralisation of PGP by daily treatment with PGP antibodies or the PGP antagonist, RTR, led to a significant reduction in the DAI and reduced shortening of the colon. Moreover, PGP neutralisation led to a significant reduction in infiltrating neutrophils in the intestine of DSS-treated mice.

PGP induces the release of both CXCL8 and MMP9 of human PMNs in vitro, ${ }^{20} 35$ and an increase in CXCL1 was seen on N-Ac-PGP-mediated neutrophilic infiltration in the lung. ${ }^{23}$ Interestingly, we found that the DSS-induced elevation of both CXCL1 and CXCL2 in the intestine was reduced by PGP neutralisation. Also, PGP neutralisation in DSS-induced colitis led to a significant reduction in elevated intestinal MMP9 levels. This suggests that PGP-induced activation of neutrophils, reflected by increased CXCL1, CXCL2 and MMP9 levels, also occurs in the intestine. A more profound activation of neutrophils by $\mathrm{N}$-Ac-PGP in the local intestinal micro environment of patients with IBD or DSS-treated mice may explain the beneficial effect of PGP neutralisation on intestinal inflammation in IBD.

In conclusion, both the proteolytic cascade that generates PGP from collagen and (N-Ac-)PGP itself are present in the intestine (figure 7). (N-Ac-)PGP-guided neutrophilic infiltration, either by a direct chemotactic effect on circulating neutrophils or activation of resident immune cells, plays an important role in intestinal inflammation. This indicates a vicious circle of neutrophil-mediated intestinal collagen proteolysis and subsequent PGP-guided infiltration of neutrophils in the intestine, and opens up new approaches to the treatment of sustained inflammation in IBD. 

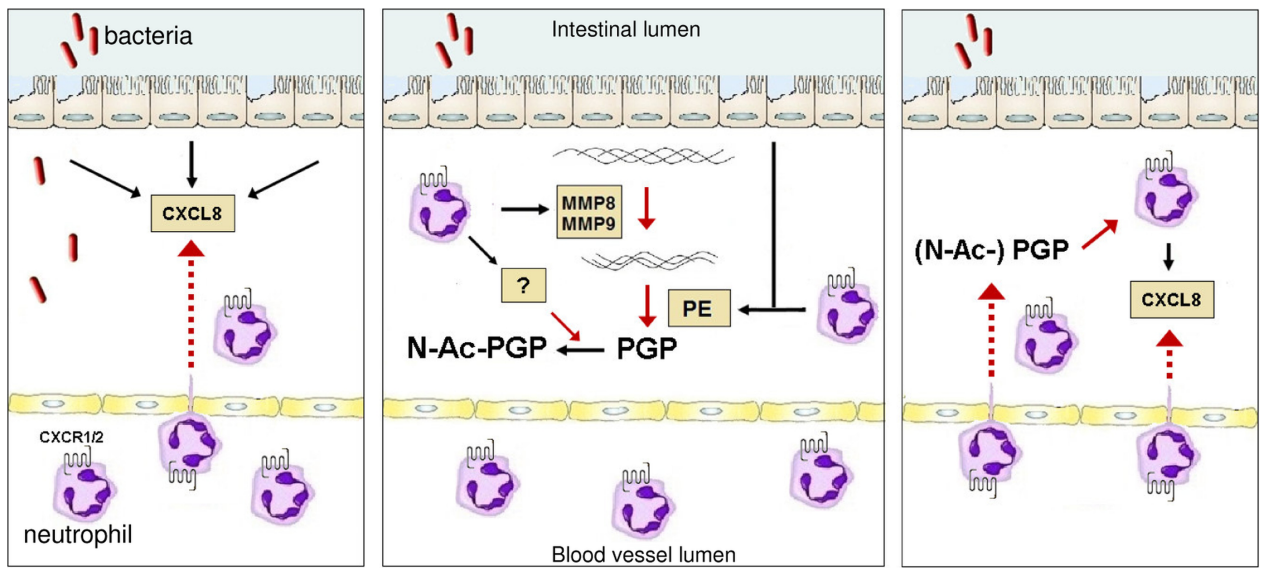

Figure 7 In inflammatory bowel disease (IBD), intestinal damage by proteases indicates a vicious circle of events leading to proline-glycineproline (PGP)-induced neutrophilic transmigration. In IBD, triggering of the epithelial layer by bacteria or their products leads to extensive release of CXCL8 by epithelial cells and subsequent CXCL8-CXCR1/2-mediated transmigration of neutrophils from the circulation (left panel). Invading neutrophils produce large amounts of matrix metalloproteinase (MMP) 8 and MMP9, which proteolytically cleave collagen into small fragments. These collagen fragments are further cleaved to the tripeptide, PGP, by epithelial- and neutrophil-derived prolyl endopeptidase (PE) (middle panel). PGP is N-terminally acetylated by an as yet unknown enzyme that is at least produced by neutrophils. (N-Ac-)PGP causes neutrophil chemotaxis and stimulates recruited neutrophils to produce CXCL8 (right panel), leading to a self-maintaining chronic situation.

\section{Author affiliations}

${ }^{1}$ Division of Pharmacology, Faculty of Science, Utrecht Institute for Pharmaceutical Sciences, Utrecht University, Utrecht, The Netherlands

${ }^{2}$ Department of Gastroenterology-Hepatology, Leiden University Medical Centre, Leiden, The Netherlands

${ }^{3}$ Tytgat Institute for Liver and Intestinal Research, Academic Medical Center, University of Amsterdam, Amsterdam, The Netherlands

${ }^{4}$ Department of Chemistry, University of Alabama at Birmingham, Birmingham, Alabama, USA

${ }^{5}$ Division of Pulmonary, Allergy and Critical Care Medicine, Department of Medicine and the Lung Health Center, University of Alabama at Birmingham, Birmingham, Alabama, USA

${ }^{6}$ Division of Pharmacoepidemiology \& Clinical Pharmacology, Department of Pharmaceutical Sciences, Faculty of Science, Utrecht Institute for Pharmaceutical Sciences, Utrecht University, Utrecht, The Netherlands

${ }^{7}$ Medicinal Chemistry and Chemical Biology, Utrecht Institute for Pharmaceutical Sciences, Utrecht University, Utrecht, The Netherlands

Contributors PJK, SAO, GF and ADK, study concept and design; PJK, SAO, SB, SCW, MAR, CWJ and PLJ, acquisition and analysis of data; PJK and ADK, writing of the manuscript; HWV, AAteV, JEB, JG, RWS and JAWK, technical and material support; MEM, PAJH, HWV, JEB, critical revision of the manuscript for important intellectual content; PLJ, JEB, GF and ADK, obtaining funding; ADK, study supervision.

Funding This study was performed within the framework of Dutch Top Institute Pharma (T1.103) and was supported in part by grants from the National Heart, Lung and Blood Institute (HL07783, HL90999 and HL087824 to JEB) and the Cystic Fibrosis Foundation (R464-CR11 to PL)). MAR was supported by a Mosaic grant from the Netherlands Organization for Scientific Research (017.008.029). The content is solely the responsibility of the authors and does not necessarily represent the official views of the National Heart, Lung and Blood Institute or the NIH.

\section{Competing interests None.}

Ethics approval The medical ethics committee of the Leiden University Medical Centre approved the intestinal tissue samples study, which complied with the Helsinki Declaration. All protocols using fresh peripheral blood were approved by the local ethics committees of the Amsterdam Medical Centre and Utrecht Medical Centre.

\section{Patient consent Obtained.}

Provenance and peer review Not commissioned; externally peer reviewed.

Open Access This is an Open Access article distributed in accordance with the Creative Commons Attribution Non Commercial (CC BY-NC 3.0) license, which permits others to distribute, remix, adapt, build upon this work non-commercially, and license their derivative works on different terms, provided the original work is properly cited and the use is non-commercial. See: http://creativecommons.org/ licenses/by-nc/3.0/

\section{REFERENCES}

1 Luster AD. Chemokines-chemotactic cytokines that mediate inflammation. The $N$ Engl J Med 1998;338:436-45.

2 Kobayashi Y. The role of chemokines in neutrophil biology. Front Biosci 2008;13:2400-7.

3 Viola A, Luster AD. Chemokines and their receptors: drug targets in immunity and inflammation. Ann Rev Pharmacol Toxicol 2008:48:171-97.

4 Laskin DL, Kimura T, Sakakibara S, et al. Chemotactic activity of collagen-like polypeptides for human peripheral blood neutrophils. J Leukoc Biol 1986;39:255-66.

5 Weathington NM, van Houwelingen $A H$, Noerager BD, et al. A novel peptide CXCR ligand derived from extracellular matrix degradation during airway inflammation. Nat Med 2006;12:317-23.

6 Gaggar A, Jackson PL, Noerager BD, et al. A novel proteolytic cascade generates an extracellular matrix-derived chemoattractant in chronic neutrophilic inflammation. J Immunol 2008;180:5662-9.

7 Haddox JL, Pfister RR, Muccio DD, et al. Bioactivity of peptide analogs of the neutrophil chemoattractant, N-acetyl-proline-glycine-proline. Invest Ophthalmol Vis Sci 1999:40:2427-9.

8 O'Reilly $\mathrm{P}$, Jackson PL, Noerager B, et al. N-alpha-PGP and PGP, potential biomarkers and therapeutic targets for COPD. Respir Res 2009;10:38.

9 Rowe SM, Jackson PL, Liu G, et al. Potential role of high-mobility group box 1 in cystic fibrosis airway disease. Am J Respir Crit Care Med 2008;178:822-31.

10 Chin AC, Parkos CA. Neutrophil transepithelial migration and epithelial barrier function in IBD: potential targets for inhibiting neutrophil trafficking. Ann N Y Acad Sci 2006;1072:276-87.

11 Atreya R, Neurath MF. Chemokines in inflammatory bowel diseases. Dig Dis 2010;28:386-94

12 Koelink PJ, Overbeek SA, Braber S, et al. Targeting chemokine receptors in chronic inflammatory diseases: An extensive review. Pharmacol Ther 2012;133:1-18.

13 Naito Y, Yoshikawa T. Role of matrix metalloproteinases in inflammatory bowel disease. Mol Aspects Med 2005;26:379-90.

14 Szeltner Z, Polgar L. Structure, function and biological relevance of prolyl oligopeptidase. Curr Protein Pept Sci 2008:9:96-107.

15 Santana A, Medina C, Paz-Cabrera MC, et al. Attenuation of dextran sodium sulphate induced colitis in matrix metalloproteinase-9 deficient mice. World J Gastroenterol 2006;12:6464-72.

16 Naito $Y$, Takagi T, Kuroda $M$, et al. An orally active matrix metalloproteinase inhibitor, 0N0-4817, reduces dextran sulfate sodium-induced colitis in mice. Inflamm Res 2004;53:462-8.

17 Bento AF, Leite DF, Claudino RF, et al. The selective nonpeptide CXCR2 antagonist SB225002 ameliorates acute experimental colitis in mice. J Leukoc Biol 2008:84:1213-21.

18 Buanne P, Di Carlo E, Caputi L, et al. Crucial pathophysiological role of CXCR2 in experimental ulcerative colitis in mice. J Leukocyte Biol 2007:82:1239-46.

19 Meijer MJ, Mieremet-Ooms MA, van der Zon AM, et al. Increased mucosal matrix metalloproteinase-1, $-2,-3$ and -9 activity in patients with inflammatory bowel disease and the relation with Crohn's disease phenotype. Dig Liver Dis 2007:39:733-9. 
20 Overbeek SA, Henricks PA, Srienc Al, et al. N-acetylated Proline-Glycine-Proline induced $\mathrm{G}$-protein dependent chemotaxis of neutrophils is independent of CXCL8 release. Eur J Pharmacol 2011;668:428-34.

21 Mihara K, Smit MJ, Krajnc-Franken M, et al. Human CXCR2 (hCXCR2) takes over functionalities of its murine homolog in hCXCR2 knockin mice. Eur J Immunol 2005;35:2573-82

22 Koelink PJ, Robanus-Maandag EC, Devilee P, et al. 5-Aminosalicylic acid inhibits colitis-associated but not sporadic colorectal neoplasia in a novel conditional Apc mouse model. Carcinogenesis 2009;30:1217-24.

23 Braber S, Overbeek SA, Koelink PJ, et al. CXCR2 antagonists block the $\mathrm{N}$-Ac-PGP-induced neutrophil influx in the airways of mice, but not the production of the chemokine CXCL1. Eur J Pharmacol 2011;668:443-9.

24 Gao Q, Meijer MJ, Kubben FJ, et al. Expression of matrix metalloproteinases-2 and -9 in intestinal tissue of patients with inflammatory bowel diseases. Dig Liver Dis 2005;37:584-92.

25 Braber S, Koelink PJ, Henricks PA, et al. Cigarette smoke-induced lung emphysema in mice is associated with prolyl endopeptidase, an enzyme involved in collagen breakdown. Am J Physiol Lung Cell Mol Physiol 2011;300:L255-65.

26 Makitalo L, Kolho KL, Karikoski R, et al. Expression profiles of matrix metalloproteinases and their inhibitors in colonic inflammation related to pediatric inflammatory bowel disease. Scan J Gastroenterol 2010;45:862-71.

27 Snelgrove RJ, Jackson PL, Hardison MT, et al. A critical role for LTA4H in limiting chronic pulmonary neutrophilic inflammation. Science 2010;330:90-4.
28 O'Reilly PJ, Hardison MT, Jackson PL, et al. Neutrophils contain prolyl endopeptidase and generate the chemotactic peptide, PGP, from collagen. J Neuroimmunol 2009:217:51-4.

29 Mitsuyama K, Toyonaga A, Sasaki E, et al. IL-8 as an important chemoattractant for neutrophils in ulcerative colitis and Crohn's disease. Clin Exp Immunol 1994;96:432-6.

30 van Houwelingen $A H$, Weathington NM, Verweij $\mathrm{V}$, et al. Induction of lung emphysema is prevented by L-arginine-threonine-arginine. FASEB J 2008:22:3403-8.

31 Graham MF, Diegelmann RF, Elson CO, et al. Collagen content and types in the intestinal strictures of Crohn's disease. Gastroenterology 1988;94:257-65.

32 McCarthy DA, Rampton DS, Liu YC. Peripheral blood neutrophils in inflammatory bowel disease: morphological evidence of in vivo activation in active disease. Clin Exp Immunol 1991;86:489-93.

33 Sharma JN, Mohammed LA. The role of leukotrienes in the pathophysiology of inflammatory disorders: is there a case for revisiting leukotrienes as therapeutic targets? Inflammopharmacology 2006;14:10-16.

34 Sharon P, Stenson WF. Enhanced synthesis of leukotriene B4 by colonic mucosa in inflammatory bowel disease. Gastroenterology 1984;86:453-60

$35 \mathrm{Xu} \mathrm{X}$, Jackson PL, Tanner S, et al. A self-propagating matrix metalloprotease-9 (MMP-9) dependent cycle of chronic neutrophilic inflammation. PloS One 2011;6: e15781. 